\title{
Für ein nuancenreicheres Bild W.G. Sebalds
}

\author{
[Contributions to a more nuanced image of W.G. Sebald] \\ http://dx.doi.org/10.11606/1982-88372135202
}

Magdalena Nowinska ${ }^{1}$

Rezension zu: Uwe Schütte $\left(\mathrm{H}_{3}.\right)$, Über W.G. Sebald. Beiträge zu einem anderen Bild des Autors. Berlin/Boston: De Gruyter, 2017.

Der im letzten Jahr erschienen Sammelband "Über W.G. Sebald" von Uwe Schütte verspricht im Untertitel "Beiträge zu einem anderen Bild des Autors". Dieses Versprechen erfüllt der Band auf zweierlei Weise: zum Einen, indem er Bereiche des Schaffens von W.G. Sebald fokussiert, die in der bisherigen Forschung entweder (noch) nicht erfasst worden sind, oder bisher eher marginal behandelt wurden, und zum Anderen, indem er dem mittlerweile konsolidierten Bild des bereits kanonischen und viel erforschten Autors Sebald neue Nuancen hinzufügen kann.

Der Sammelband versammelt 16 Texte, davon 14 Aufsätze und zwei persönliche Erinnerungstexte ehemaliger Doktoranden Sebalds. Mit diesem Band stellt Schütte sich explizit gegen die Mainstream-Kritik Sebalds, zu der er mit dem Band ein Korrektiv liefern will. Der Sammelband will "in das von der Sebald-Forschung bislang gezeichnete Bild der Autors zwischen Hagiografie, Apologie, Stilisierung und Verengung" (4) intervenieren und über den von Schütte konstatierten "engen Kreis an Fragestellungen zu den immer gleichen Themen, die man auf die Schlagwörter Trauma - Holocaust Intermedialität - Erinnerung - Melancholie reduzieren kann" (4) hinausgehen. Nicht das

\footnotetext{
1 Universidade Estadual de Campinas, Instituto de Estudos da Linguagem, Departamento de Teoria Literária, Rua Sérgio Buarque de Holanda, 571, Campinas, SP, 13083-872, Brasil. E-mail: mnowinska@posteo.org.
}

\section{(cc) BY-NC}

Pandaemonium, São Paulo, v. 21, n. 35, set.-dez. 2018, p. 202-207 
Nowinska, M. - Für ein nuancenreicheres Bild W.G. Sebalds

zentrale literarische Oevre ist dabei Thema der Aufsätze des Bandes, sondern weniger bekannte Texte Sebalds sowie bestimmte Aspekte seiner Poetologie.

Die Bemühung um eine Diversifizierung des Bildes vom Autor und Menschen W.G. Sebald wird ersichtlich nicht nur in der Auswahl der Themen der Aufsätze, sondern auch in den Biografien der versammelten Autoren, von denen einige, aber längst nicht alle dem akademischen Betrieb angehören: es finden sich unter ihnen auch Übersetzer, freie Wissenschaftler oder Verleger, sowie Quereinsteiger in den und Aussteiger aus dem akademischen Betrieb. Ebenso heterogen präsentiert sich die nationale und geografische Verteilung der deutschen, englischen und amerikanischen Autoren im Band, die größtenteils in Ländern wie England, Italien, Spanien, Taiwan oder Japan leben und arbeiten, wobei die Heterogenität und geografische Exzentrizität der Autoren gleichsam auch die eher exzentrische Position W.G. Sebalds selbst im deutschsprachigen literaturwissenschaftlichen Betrieb reflektiert.

Das Projekt ist durchaus auch persönlich gefärbt: Schütte, ein ehemaliger Doktorand W.G. Sebalds, will mit dem Band einem "vorbildhaften Lehrer" (15) eine Ehre erweisen, indem er unorthodoxe Zugänge zu seinem Werk veröffentlicht und zugleich versucht, "ein anderes Bild des Autors zu zeichnen als das von der Germanistik und Literaturkritik nicht selten produzierte Wunschportrait" (15). Es geht Schütte auch nicht nur um dem Schriftsteller Sebald, sondern auch um die Person Sebald, er zielt auf ein "differenziertes Gesamtbild" (8) beider Aspekte ab. Eine Hommage an Sebald stellt schließlich die englisch-deutsche Zweisprachigkeit des Bandes dar, die mit der doppelten Beheimatung Sebalds korrespondiert.

Die Konstruktion eines "anderen Bildes" von W.G. Sebald wird in drei Bereichen unternommen: zum Einen behandeln die Aufsätze die Entwicklung des Schriftstellers Sebald sowie seine Wirkung auf andere Autoren; zum Zweiten werfen die Autoren neue Blicke auf bereits bekannte Aspekte des Werks Sebalds; und zum Dritten wird auch ein W.G. Sebald jenseits der bekannten Prosawerke analysiert.

Der weitaus größte Teil der Texte beleuchtet die Entwicklung und die Wirkung Sebalds als Autor. Vier Aufsätze sind unveröffentlichten und nur im Archiv zugänglichen Texten Sebalds gewidmet: Melissa Etzler analysiert einen Romanentwurf Sebalds aus dem Jahr 1967, Michael Hutchins untersucht zwei unvollendete dramaturgische Versuche 
Sebalds, Uwe Schütte beschäftigt sich mit Sebalds "Kant-Projekt" aus den 1980er Jahren, einer als experimentelles Fernsehspiel gedachten Szenenreihe, und Scott Bartsch schreibt über Sebalds "Prosa-Projekt", eine Vorarbeit und Vorstufe zu den später erschienenen "Schwindel.Gefühle" und "Die Ausgewanderten". Gemeinsam ist den Aufsätzen, dass sie alle aufzeigen, dass die Juvenilia Sebalds zwar sprachlich eine Rohform der späteren Texte darstellen, jedoch in ihnen bereits zahlreiche Themen und Denkweisen Sebalds, die für sein literarisches Werk zentral sind, bereits vorhanden sind. Die von allen Autoren ausführlich zitierten archivalischen Textauszüge sind als Vergleich zum Schreiben des späteren Sebalds von großem Interesse. Zugleich zeigen auch die diversen Textformen, dass Sebalds spätere Entscheidung für Prosa als Medium nicht von vornherein zwingend war, sondern dass er durchaus auch mit anderen Formaten experimentiert hat; besonders kurios ist dabei angesichts der notorischen Technikfeindlichkeit Sebalds das Fernsehspiel.

Zum gleichen Themenbereich, aber mit anderem Schwerpunkt gehören drei Aufsätze, die Sebalds literaturkritische Schriften als eine Art poetologische Übungen analysieren. Ulrich Dronske zeigt anhand der Analyse von Sebalds Döblin-Buch die Wandlung im Denken Sebalds von radikaler und kompromissloser Kritik zu einer von Empathie bestimmten Sicht auf Geschichte. Markus Joch analysiert anhand von Sebalds kritischem Aufsatz zu Jurek Becker, wie sich Sebald als Autor durch Kritik an Autoren profiliert, die Themen behandeln, die ihm nahe lagen. Jakob Heyner schließlich untersucht "Luftkrieg und Literatur" im Zusammenhang von Sebalds Restitutionsprojekt.

Der Wirkung des Schreibens von Sebald auf zeitgenössische Autoren widmet sich schließlich der Aufsatz von Adrian West, der zwei Romane der amerikanischen Autoren Teju Cole und Ben Lerner analysiert und die Texte als misslungene Versuche ansieht, die von postmodernen Standpunkt unzeitgemäße, aber in der amerikanischen Gegenwartsliteratur ersehnte Ernsthaftigkeit der Prosa Sebalds zu emulieren.

Eine zweite Gruppe von Aufsätzen in Schüttes Band wirft neue Blicke auf bekannte Aspekte des Werks Sebalds. Anti-aufklärerische bzw. anti-rationale Tendenzen im Denken und Werk Sebalds werden in drei Texten untersucht: Sven Meyer spekuliert anhand der Präsenz von Rupert Sheldrakes Ideen in den Texten Sebalds über die Affinität Sebalds zu esoterischem Denken und inwieweit dieses für Sebald "nur" eine 
poetologische Funktion hatte oder darüber hinaus ging; Christoph Steker schreibt über die Bedeutung des Botanischen in Sebalds Werk, insbesondere mit Blick auf das unvollendete Korsika-Projekt, und darüber, inwieweit Sebald Natur als Gegensatz zur Zivilisation darstellt; Christian Hein schließlich nimmt sich Sebalds pessimistisches Konzept der Naturgeschichte der Zerstörung vor und untersucht den anti-aufklärerischen Aspekt der Sicht Sebalds auf Natur als alles verzehrende Gewalt. Die Präsenz des Antirationalen in Sebalds Werk ist an sich kein Novum, Sebalds Verfahren der Koinzidenzpoetik, wie sie von Marcel Atze definiert wurde, oder der von Claude LeviStrauss entlehnten Bricolage, seine Beschäftigung mit Dichtern wie Ernst Herbeck - dies alles sind bekannte Aspekte des Werks. Das Verdienst dieser Texte ist eher darin zu sehen, dass sie das sonst eher marginal behandelte antirationale Denken Sebalds ins Zentrum der Betrachtung rücken.

In ähnlicher Weise behandeln die beiden anderen Texte dieser Gruppe ebenfalls bereits bekannte Themen aus der Sebald-Forschung, suchen jedoch zugleich neue Aspekte zu beleuchten. Peter Schmucker beschäftigt sich mit Fragen der Intertextualität bei Sebald, einem der zentralen Features der literarischen Texte Sebalds. Anhand der intertextuellen Präsenz von Chateaubriand in diversen Texten Sebalds, und im Kontrast zu Julia Kristevas Intertextualitätsbegriff, erarbeitet Schmucker einen Intertextualitätsbegriff für Sebald, der dem Zweck der bewussten Rezeptionssteuerung und der Verrätselung der Texte dient. Durch unterschiedlich markierte intertextuelle Verweise will Sebald, so Schmucker, eine "paranoide" Haltung beim Leser hervorrufen, aufgrund derer der Leser ständig den Text auf intertextuelle Verweise hinterfragt.

Kay Wolfinger wiederum schreibt über das Allgäu als unheimliche Heimat Sebalds. Sein Text stellt einerseits eine detaillierte Analyse der Fiktionalisierungen des Allgäus, die Sebald in seinen Texten vorgenommen hat, und zeigt andererseits zugleich, dass Sebalds reales Verhältnis zu seiner Heimat wesentlich entspannter war, als die Texte suggerieren. Das in vielen Texten, auch Essays, literarisierte Allgäu war für Sebald, so Wolfinger, ein "Argumentationsort für konservative Zivilisationskritik", ein dystopischer Ort, der dem Autor Sebald ein Orts- und Figurenarsenal zur Verfügung stellte und als Movens der Fiktion diente. 
Drei Texte des Bandes widmen sich schließlich einem W.G. Sebald jenseits der bekannten Prosawerke, nämlich Sebald dem Lyriker und Sebald dem Akademiker, zwei Bereiche, die in der im überwältigenden Maße auf das Prosawerk konzentrierten SebaldForschung ohne Zweifel eine bisher marginale Rolle gespielt haben. Axel Englund, Übersetzer von Sebald ins Schwedische, widmet sich der Lyrik Sebalds, insbesondere der Mikropoesie der zwei Bände "For Years Now" (2001) und des postumen "Unerzählt" (2004). Die Lyrik Sebalds wird, so Englund, gegenüber der Prosa in der Kritik vernachlässigt, und das obwohl Sebald über 40 Jahre lang mehr oder weniger regelmäßig Gedichte veröffentlicht hat. Dies widerspiegelt dabei Sebalds eigene Bedenken gegenüber der Lyrik, gegen die er als "Kritzelei" ambivalent eingestellt war und letztendlich der Prosa den Vorzug gab. Englund hebt hervor, dass Sebalds Lyrik nicht mit der gleichen Optik gelesen werden kann wie die Prosawerke, insbesondere Austerlitz: die Themen und Verfahren unterscheiden sich grundsätzlich. Während die Prosa Fragen der Geschichte und Erinnerung im Zentrum hat, beschäftigen sich die Gedichte Sebalds mit Fragen der Subjektivität und der Wahrnehmung.

Beschlossen wird der Band durch zwei persönliche Erinnerungstexte ehemaliger Doktoranden Sebalds, Hommagen an den "außergewöhnlichen Hochschullehrer" Sebald. Ralf Jeutter porträtiert Sebald als "einen einzigartigen Mann" und analysiert einige Aspekte der Persönlichkeit Sebalds. Florian Radvan beschreibt Sebald als Lehrer sui generis, wobei er sich auf Sebalds Fähigkeit konzentriert, Literatur zu vermitteln. Das Verdienst dieser persönlichen Texte in dem Band ist vor allem darin zu sehen, dass sie daran erinnern, dass die akademische Tätigkeit bis zu seinem Debüt als Schriftsteller die eigentliche Berufung Sebalds gewesen ist, und auch noch danach einen zentralen Aspekt des Lebens Sebalds darstellte.

Uwe Schütte ist bereits ein bekannter Name in der Sebald-Forschung, vor allem auch durch seine Ansätze, weniger diskutierte Aspekte des Autors zu beleuchten sowie differenzierte Zugänge zum Werk Sebalds aufzuzeigen. Das Buch, das dieser Art von Ansatz verpflichtet ist, erscheint wenige Jahre nach dem Boom um die Werke des Autors, der auf dessen Tod gefolgt war. Angesichts der sehr rasanten Kanonisierung W.G. Sebalds als Gegenwartsautor ist es vielleicht nicht überraschend, dass auch erste Gegenstimmen zu dieser Kanonisierung früh aufkommen, wobei der von Schütte 
Nowinska, M. - Für ein nuancenreicheres Bild W.G. Sebalds

herausgegebene Band sich nicht nur als kritischer Beitrag zu W.G. Sebald, sondern vor allem als Kritik an einer als einseitig verstandenen akademischen Rezeption Sebalds versteht.

Schüttes Ansatz ist freilich nicht ohne innere Unstimmigkeiten, etwa wenn er einerseits die "akademische Approbation" Sebalds in "repräsentativen Handbüchern" (5) kritisiert, andererseits aber selbst an genau solchen Handbüchern sich beteiligt (z.B. am "W.G. Sebald-Handbuch" vom Metzler Verlag), oder wenn er etwa das Unverständnis beklagt, auf das bestimmte, vor allem polemische Texte Sebalds in der germanistischen Kritik gestoßen sind, dann aber wiederum den allzu großen Konsens in der gleichen Kritik beklagt - der Verdacht drängt sich auf, nicht Konsens oder Dissens seien hier die Angriffspunkte, sondern die Institution der Germanistik an sich.

Jedoch stören diese kleinen Dissonanzen nicht das Verdienst des Bandes, der seinem Anspruch gerecht wird, dem Bild von Sebald neue Facetten zu verleihen. Die Themen und Autoren reflektieren diesen Ansatz, und eines der Verdienste ist sicherlich eine Aufwertung der Auslandsgermanistik, eine Haltung gegenüber geographisch und institutionell marginalisierten Positionen, die zweifellos dem Sinne Sebalds entspricht. Durch seine exzentrische Schwerpunktsetzung fügt der Band somit dem etablierten Bild W.G. Sebalds neue und wichtige Nuancen hinzu.

Recebido em 08 de maio de 2018 Aceito em 17 de maio de 2018 